\title{
Rank-based inference for the accelerated failure time model in the presence of interval censored data
}

\begin{abstract}
Semiparametric analysis and rank-based inference for the accelerated failure time model are complicated in the presence of interval censored data. The main difficulty with the existing rank-based methods is that they involve estimating functions with the possibility of multiple roots. In this paper a class of asymptotically normal rank estimators is developed which can be aquired via linear programming for estimating the parameters of the model, and a two-step iterative algorithm is introduce for solving the estimating equations. The proposed inference procedures are assessed through a real example.
\end{abstract}

Keyword: Accelerated failure time model; Interval censored data; Rank-based inference; Semiparametric analysis 